\title{
Philosophiques
}

\section{Le moi-substance : une interprétation de l'" analyse du morceau de cire " de la seconde Méditation}

\section{Denis Sauvé}

Volume 16, numéro 1, printemps 1989

URI : https://id.erudit.org/iderudit/027065ar

DOI : https://doi.org/10.7202/027065ar

Aller au sommaire du numéro

Éditeur(s)

Société de philosophie du Québec

ISSN

0316-2923 (imprimé)

1492-1391 (numérique)

Découvrir la revue

Citer cet article

Sauvé, D. (1989). Le moi-substance : une interprétation de l'« analyse du morceau de cire » de la seconde Méditation. Philosophiques, 16(1), 73-108. https://doi.org/10.7202/027065ar
Résumé de l'article

Descartes semble s'être donné plus d'un but dans le passage du morceau de cire de la seconde Méditation. L’un est de dire quelles propriétés des corps sont claires et distinctes ou appartiennent à leur essence. Un autre est de montrer en quoi le moi pensant est « mieux connu » que les choses matérielles bien que, contrairement aux corps, il ne puisse être représenté par les sens ou à l'aide de l'imagination. Il fait également des remarques importantes à propos du rôle de l'entendement, par opposition à celui des sens, dans notre connaissance aussi bien de l'esprit que des corps. La question traitée dans ce qui suit est celle de savoir quel est son but premier. Celui-ci, d'après l'inter- prétation que je vais défendre, a égard non pas à notre conception des corps mais, comme dans le reste de cette Méditation, à celle de l'esprit considéré comme une substance pensante. 
PHILOSOPHIQUeS, Vol. XVI, Numéro 1, Printemps 1989

\title{
LE MOI-SUBSTANCE : UNE INTERPRÉTATION DE L'«ANALYSE DU MORCEAU DE CIRE » DE LA SECONDE MÉDITATION
}

\author{
par Denis Sauvé
}

\begin{abstract}
RÉSUMÉ. Descartes semble s'être donné plus d'un but dans le passage du morceau de cire de la seconde Méditation. L'un est de dire quelles propriétés des corps sont claires et distinctes ou appartiennent à leur essence. Un autre est de montrer en quoi le moi pensant est «mieux connu » que les choses matér ielles bien que, contrairement aux corps, il ne puisse être représenté par les sens ou à l'aide de l'imagination. Il fait également des remarques importantes à propos du rôle de l'entendement, par opposition à celui des sens, dans notre connaissance aussi bien de l'esprit que des corps. La question traitée dans ce qui suit est celle de savoir quel est son but premier. Celui-ci, d'après l'interprétation que je vais défendre, a égard non pas à notre conception des corps mais, comme dans le reste de cette Méditation, à celle de l'esprit considéré comme une substance pensante.
\end{abstract}

ABSTRACT. Descartes seems to have several purposes in mind in the well known " piece of wax passage " of the second Meditation. One is to state which properties of material things are clear and distinct, or belong to their essence. Another is to show that the thinking self is "better known" than material things, although, unlike bodies, it is not something which falls under sense or imagination. In addition, he also makes some important points concerning the role of the understanding, as opposed to the senses, in our knowledge of the mind and of bodies. The question I wish to pose in this paper is: What is Descartes' main goal ? According to the interpretation I will defend, his first concern relates not to our conception of bodies but, as in the rest of this Meditation, to our conception of the mind as a thinking substance.

Le texte de la seconde Méditation se divise en deux parties relativement indépendantes : Descartes entreprend dans la première 
de montrer que l'essence de l'esprit est d'être seulement une chose qui pense; ensuite, dans la deuxième, où il invoque son célèbre exemple du morceau de cire, il dit, entre autres, en quoi consiste notre idée des choses matérielles. Ce dernier passage, d'après l'interprétation courante, traite de la nature de la matière : l'exemple du morceau de cire est censé montrer que l'idée claire et distincte que nous avons d'un corps est l'idée de quelque chose d'étendu. Il semble que Descartes veuille tirer aussi d'autres conclusions de son "analyse », entre autres que l'esprit est plus « facile » à connaître que les corps (ou, d'après le titre latin de la Méditation, qu'il est “mieux connu» qu'eux), et il soutient que la connaissance des corps, quand elle n'est pas obscure et confuse, dépend de l'entendement et non des sens ou de l'imagination. Mais bien qu'en ce point des Méditations nous ne sachions pas encore si ceux-ci existent et s'ils sont réellement tels que nous les concevons, le but principal du passage est de mettre en évidence cette idée que nous avons de leur essence.

Je propose dans ce qui suit une interprétation qui diffère sur un point important de l'interprétation courante. Descartes, il me semble, veut dire ici quelque chose non pas seulement au sujet de la nature du morceau de cire mais aussi, et surtout, au sujet de sa représentation ( claire et distincte 》) comme substance. Les remarques qu'il fait à propos de l'essence des choses matérielles ont une importance fondamentale, sans doute, si on les replace dans le contexte de l'ensemble des Méditations: elles sont reprises et développées dans les Méditations ultérieures, en particulier dans la troisième (où il jette les bases de la distinction entre qualités primaires et secondaires), la cinquième (intitulée « De l'essence des choses matérielles (...) ») et dans la sixième (où il démontre la "distinction réelle» de l'esprit et du corps). Mais, considéré seulement dans le contexte de cette seconde Méditation, on saisit mieux la signification et la structure du passage du morceau de cire si on suppose que c'est d'abord la substantialité des corps qui intéresse ici Descartes. Si cette façon de l'interpréter est exacte, on peut comprendre aussi que le propos qui sous-tend le passage vise en réalité au moins autant la substantialité du moi pensant que celle des corps: Descartes, comme je le dirai plus loin, veut défendre à cet endroit l'idée, qu'il place au centre de sa métaphysique, d'après laquelle l'esprit est une substance au même titre que les 
choses matérielles et non pas, comme pour Aristote et les scolastiques, une «forme» du corps.

J'exposerai d'abord l'interprétation communément admise du texte (et certaines de ses variantes) pour la discuter et en faire la critique. Ensuite, je défendrai une autre interprétation. ${ }^{1}$

Descartes introduit le passage du morceau de cire en le présentant comme une réponse à une objection qu'est susceptible de lui faire son lecteur. Dans la première partie de la Méditation, il a montré d'abord par le Cogito qu'il (son propre moi ou son esprit) est une chose qui existe réellement et, ensuite, qu'il a une idée de ce moi seulement comme d'une chose qui pense. Mais cette connaissance qu'il a de son esprit, il ne l'a obtenue, dit-il, ni par les sens, ni à l'aide de l'imagination: "[La] notion et connaissance de moimême, ainsi précisément prise, ne dépend point des choses dont l'existence ne m'est pas encore connue; ni par conséquent (...) d'aucunes de celles qui sont feintes et inventées par l'imagination. » ${ }^{2}$ Il est même nécessaire de " détourner son esprit de cette façon de concevoir [qui a recours à l'imagination], afin qu'il puisse luimême reconnaître bien distinctement sa nature ». ${ }^{3}$ Pourtant, et c'est ici qu'on pourrait lui faire une objection, on considère habituellement que toute connaissance doit se rapporter d'une façon ou d'une autre, directement ou indirectement, à ce qui est donné au moyen des sens (à des perceptions visuelles, auditives, tactiles, etc.) et que, pour cette raison, la meilleure connaissance que l'on ait de quoi que ce soit est celle que l'on a des corps; comme le disaient les scolastiques, nibil est in intellectu quod prius non fuerit in sensu. Et si toute connaissance doit nécessairement débuter par le témoignage des sens et, au moins initialement, prendre pour objet les choses matérielles, alors l'idée d'une connaissance du moi s'avère pour le moins problématique dans la mesure

1. Les références aux écrits de Descartes, dans ce qui suit, renvoient, pour le texte latin, à l'édition publiée par C. ADam et P. Tannery (CEuvres de Descartes, Paris : Vrin, 1973) et, pour la traducrion française, à l'édition publiée par F. ALQUIÉ (Euvres philosophiques, Paris : Garnier, 1963-1973). La première édition est signalée par les lettres AT, la deuxième par la lettre $A$.

2. AT VII, 27-28; A II, 420 .

3. AT VII, $28 ; A$ II, 420 . 
où, justement, celui-ci n'est pas quelque chose qui peut être perçu à travers les sens ou représenté par des images au moyen de l'imagination. Descartes écrit (c'est le début du passage) : il semble, et je ne peux m'empêcher de croire, "que les choses corporelles, dont les images se forment par ma pensée, et qui tombent sous le sens, ne soient plus distinctement connues que cette je ne sais quelle partie de moi-même qui ne tombe point sous l'imagination ${ }^{4}$, c'est-à-dire mon moi ou mon esprit. Ainsi, d'après l'objection, on ne peut connaître la nature de l'esprit pas plus, d'ailleurs, que l'existence de Dieu, puisque ni l'âme ni Dieu ne sont des choses qui tombent sous le sens; ou, du moins, on ne peut les connaître aussi bien ou, peut-être, aussi directement et parfaitement, que l'on peut connaître les choses matérielles.

L'objection est celle que ferait un philosophe de la tradition aristotélicienne. Dans le Discours, en se référant à l'adage scolastique, Descartes remarque que « (...) même les philosophes tiennent pour maxime, dans les écoles, qu'il n'y a rien dans l'entendement qui n'ait premièrement été dans le sens, où toutefois il est certain que les idées de Dieu et de l'âme n'ont jamais été. "s Pour y répondre, il va essayer de montrer que non seulement dans le cas de l'âme, mais même dans celui des choses matérielles, on ne peut parvenir à une véritable connaissance des choses au moyen uniquement des sens ou de l'imagination. Cette connaissance, aussi bien de l'âme que des corps, y compris de leur essence, n'est possible selon lui qu’à l'aide de l'entendement. Comme il le dit au même endroit dans le Discours, « (...) ni notre imagination, ni nos sens ne nous sauraient jamais assurer d'aucune chose, si notre entendement n'y intervient. ${ }^{6}$

«Commençons", dit-il, «par la considération des choses les plus communes», par exemple de ce morceau de cire. Celui-ci possède, suivant l'opinion ordinaire, toutes les propriétés «qui peuvent distinctement faire connaître un corps (...) ${ }^{7}$ : il a une certaine odeur, une saveur, une couleur, une forme particulière et une grandeur; il est dur et froid au toucher et, lorsqu'on le frappe,

\footnotetext{
4. AT VII, 29; A II, 422.

5. A I, 609 .

6. Ibid.

7. AT VII, 30 ; A II, 423
} 
il produit un son. Mais qu'arrive-t-il si on l'approche du feu? Son odeur disparaît, sa couleur change, sa grandeur augmente, il prend une autre forme, il devient chaud au toucher et il ne donne plus aucun son quand on le frappe. Néanmoins, malgré tous ces changements, personne ne nie que le morceau de cire demeure. Mais , demande Descartes, "[q]u'est-ce donc que l'on connaissait en ce morceau de cire avec tant de distinction? "Et il répond: «Certes ce ne peut être rien de tout ce que j'y ai remarqué par l'entremise des sens, puisque toutes les choses qui tombaient sous le goût, ou l'odorat, ou la vue, ou l'attouchement, ou l'ouie se trouvent changées, et cependant la même cire demeure [remanet cera]. ${ }^{8}$

On trouve dans ce passage une inférence dont le sens, pour le moins, n’apparaît pas de façon évidente. Descartes veut déduire, semble-t-il, de ce que toutes les propriétés du corps «remarquées par l'entremise des sens » ont changé, qu'aucune de ces propriétés, contrairement à ce que suppose l'opinion ordinaire, ne nous fait connaître réellement ce qu'il est: puisque ces propriétés ont changé, elles ne donnent pas une connaissance distincte du morceau de cire. Comment comprendre son raisonnement?

Une interprétation partagée par plusieurs commentateurs des Méditations est la suivante. ${ }^{9}$ Dans la suite du texte, on lit que les propriétés sensibles du morceau de cire ne font pas connaître distinctement ce qu'il est parce que, en réalité, la cire n'est «ni cette agréable odeur des fleurs, ni cette blancheur, ni cette figure, ni ce son, mais seulement un corps (...). » ${ }^{10}$ Plus exactement, comme on le lit un peu après, l'idée claire et distincte que renferme la représentation du morceau de cire et qui va servir à définir son essence est celle seulement de quelque chose d'étendu (dans les trois dimensions), de «flexible 》 (susceptible de recevoir différentes formes) et de «muable» (capable de mouvement). Aucune de ses

8. AT VII, 30 ; A II, 424 .

9. Cf., par exemple, Margaret WILSON, Descartes (Londres : Rourledge and Kegan Paul, 1978, édition révisée 1982), p. 79 ; Geneviève Rodis-Lewis, Descartes (Paris : Librairie Générale Française, 1984), p. 241 ; L.J. BECK, The Metaphysics of Descartes. A Study of the "Meditations» (Oxford: Clarendon Press, 1965), p. 102. Bernard Williams, par contre, Descartes: The Project of Pure Enquiry (Atlantic Highlands, N. J. : Humanities Press, 1978), rejetre cetre interprétation (cf. p. 213 et suiv.).

10. AT VII, 30-31; A II, 424. 
autres propriétés telles que l'odeur, le goût, la couleur, le froid ou la chaleur et les autres qualités tactiles n'appartient à son essence. La distinction entre les deux types de propriétés, les propriétés sensibles et les propriétés essentielles ou constitutives du morceau de cire, s'explique apparemment par un principe (implicite) se formulant: "Les propriétés changeantes d'un corps ne sont pas des propriétés qui appartiennent à sa nature (elles n'en donnent pas une idée claire et distincte) ; seules ses propriétés permanentes peuvent être considérées comme étant comprises dans son essence. » À l'aide de ce principe, on pourrait interpréter comme suit le raisonnement: parce que les propriétés sensibles de la cire ont toutes changé, elles ne font pas partie de son essence; l'extension, la flexibilité et la mutabilité, au contraire, sont des propriétés qui ont demeuré, et c'est pourquoi on peut dire qu'elles constituent l'essence du morcau de cire (et qu'elles en donnent une connaissance distincte).

Est-ce bien le raisonnement de Descartes? Prenons la propriété d'avoir une odeur. Le morceau de cire a perdu son odeur quand il a été approché du feu, et on pourrait dire effectivement suivant le principe que l'odeur des fleurs qu'il avait auparavant ne fait pas partie de son essence puisqu'il s'agit seulement d'un propriété «changeante » du corps. De même, parce qu'il ne produit plus un son quand on le frappe, il ne possède pas par nature la propriété de donner un son. Si on passe à la couleur, on peut dire que la blancheur n'est pas non plus une de ses propriétés invariantes parce qu'au début il était blanc et qu'après avoir été réchauffé il a changé de couleur et il est devenu (disons) jaune, de sorte qu'il n'est par essence ni blanc ni jaune. Mais la difficulté est que ce critère ne permet pas d'éliminer toutes les propriétés qui devraient en être exclues. Par exemple, le corps a perdu sa couleur blanche pour devenir ensuite jaune, mais à travers ce changement il a conservé la propriété d'avoir une couleur; et s'il était froid pour devenir ensuite chaud, il a toujours, après le changement, la propriété d'avoir une température. Être coloré et avoir une température représentent des traits permanents du morceau de cire et il est clair pourtant qu'ils n'appartiennent pas à la nature du corps. ${ }^{11}$

11. Bernard Williams fait cetre objection à l'interprétation habituelle du texte (Descartes: The Project of Pure Enquiry, p. 217 et 218). 
On pourrait suggérer peut-être une autre façon de formuler le principe, pour déterminer en quoi consiste l'essence des choses matérielles, qui serait celle-ci: "si le corps perd une de ses propriétés particulières (comme avoir l'odeur des fleurs) ou si une de ces propriétés (par exemple être blanc) est remplacée par une autre d'un même type (être jaune), alors la propriété générale correspondante (avoir une odeur ou une couleur) n'appartient pas à son essence ». D'après ce critère, le morceau de cire ne possède par nature aucune de ces propriétés : « avoir une odeur » (puisqu'il a perdu l'odeur des fleurs), "avoir une température " (il est passé du froid au chaud), "avoir une couleur» (il a changé de la couleur blanche à la couleur jaune) et "produire un son" (il a perdu en se liquéfiant sa capacité de donner un son quand on le frappe). On pourrait donc exclure des propriétés que n'éliminait pas le premier principe. La difficulté cependant, à l'inverse de la première, est que l'on exclut maintenant trop de propriétés. Après avoir été réchauffé, le corps a changé de forme; il avait, disons, une forme cylindrique et il a acquis une autre forme en devenant liquide, ce qui implique qu'il ne possède pas la propriété générale correspondante «avoir une forme», c'est-à-dire «être flexible». De façon similaire, il était au début immobile et il est maintenant (supposons) en mouvement, de sorte que d'après le principe il n'est pas non plus «muable ». Pourtant, on devrait compter la flexibilité et la mutabilité au nombre de ses propriétés essentielles. D'autre part, il semble que l'on doive même exclure l'extension : en se liquéfiant le corps a changé de longueur (il avait auparavant la propriété particulière d'avoir une longueur $x$ et il a maintenant une longueur $y$ ), de sorte qu'il ne possède pas la propriété générale «avoir une longueur» ou, pour la même raison, "avoir une profondeur» (et «avoir une largeur »), et il s'ensuivrait qu'il n’est pas non plus étendu.

On peut douter qu'il existe un autre critère relatif à des traits " permanents » des corps (ou faisant appel à une distinction entre des propriétés "particulières» et des propriétés "générales») dont résulteraient exactement les trois propriétés (extension, flexibilité et mutabilité) dans lesquelles Descartes voit des propriétés pouvant donner une connaissance distincte du morceau de cire. On pourrait envisager une autre interprétation. Dans la deuxième partie des Principes (article 4), on trouve un passage, parallèle, semble-t-il, à celui de la seconde Méditation, qui suggère une façon 
différente de se représenter le raisonnement concluant que ce n'est pas par leurs propriétés sensibles que nous pouvons obtenir une connaissance distincte des corps. Il s'agit dans ce passage de montrer «que la nature de la matière, ou du corps pris en général, ne consiste point en ce qu'il est une chose dure, ou pesante, ou colorée, ou qui touche nos sens de quelque autre façon, mais seulement en ce qu'il est une substance étendue en longueur, largeur et profondeur. ${ }^{12} \mathrm{Il}$ propose à cet endroit ce que l'on pourrait considérer comme une sorte de Gedankenexperiment. Prenons, dit-il, par exemple, une propriété sensible qu'ont certains corps telle que la dureté : « (...) nous n'en connaissons autre chose, par le moyen de l'attouchement, sinon que les parties des corps durs résistent au mouvement de nos mains lorsqu'elles les rencontrent (...). » ${ }^{13}$ Mais on pourrait imaginer une situation comme la suivante: si «toutes les fois que nous portons nos mains vers quelque part, les corps qui sont en cet endroit se retiraient aussi vite comme elles en approchent, il est certain que nous ne sentirions jamais de dureté ; et néanmoins nous n'avons aucune raison qui nous puisse faire croire que les corps qui se retireraient de cette sorte perdissent pour cela ce qui les fait corps [à savoir leur extension]. " ${ }^{14}$ Il semble qu'une même idée, ou une idée similaire, soit développée plus longuement un peu plus loin à l'article 11 ; il écrit : « [pour] mieux discerner quelle est la véritable idée que nous avons du corps nous [pouvons prendre] pour exemple une pierre et en [enlever] tout ce que nous saurons ne point appartenir à la nature du corps. ${ }^{15}$ Ainsi, on peut ôter de la pierre la dureté, "parce que, si on [la] réduisait (...) en poudre, elle n'aurait plus de dureté, et ne laisserait pas pour cela d'être un corps (...).» On pourrait faire de même pour les autres propriétés comme la couleur, la pesanteur et la température : «[ô]tons (...) aussi [du corps] la couleur, parce que nous avons pu voir quelquefois des pierres si transparentes qu'elles n'avaient point de couleur; ôtonsen la pesanteur, parce que nous voyons que le feu, quoiqu'il soit très léger, ne laisse pas d'être un corps; ôtons-en le froid, la chaleur, et toutes les autres qualités de ce genre, parce que nous ne

12. AT VIII, 42 ; A III, 149.

13. Ibid.

14. Ibid.

15. AT VIII, $46 ;$ A III, 156. 
pensons point qu'elles soient dans la pierre, ou bien que cette pierre change de nature parce qu'elle nous semble tantôt chaude et tantôt froide. » ${ }^{16} S$ 'il est possible d'imaginer une situation telle que l'on pourrait ne jamais avoir l'occasion de percevoir une des qualités sensibles des corps (comme avoir l'impression de la dureté, parce qu'ils s'éloignent toutes les fois qu'on tente de les toucher), ou si on peut en concevoir un seul qui soit dépourvu de l'une de ces propriétés (comme avoir une couleur ou une température), alors on peut dire que celles-ci ne sont pas des propriétés constitutives des corps.

Le rapprochement entre le texte de la deuxième Méditation et ces passages des $P$ rincipes permettrait de comprendre pourquoi Descartes considère que seules l'extention, la flexibilité et la mutabilité appartiennent à la nature de la matière. ${ }^{17}$ Dans la deuxième Méditation, on lit: "Considérons [le morceau de cire] attentivement, et éloignant toutes les choses qui [ne lui] appartiennent point (...), voyons ce qui reste. Certes il ne demeure [supersit] rien que quelque chose d'étendu, de flexible et de muable. » ${ }^{18}$ On doit éloigner « toutes les choses qui n'appartiennent point [au morceau de cire] » et voir ensuite ce qui reste, c'est-à-dire éloigner toutes ces propriétés qui, comme dans les exemples des Principes, pourraient être retranchées du corps sans qu'il cesse d'exister ou, plus précisément, toutes les qualités qui pourraient être enlevées de l'idée du corps sans que soit supprimée l'idée (complexe) que l'on en avait initialement. Pour employer la terminologie de Kant, l'«expérience de pensée» des Principes revient à faire l'«analyse» (la décomposition) du concept d'un corps dans le but de déterminer quels éléments lui sont attachés nécessairement et lesquels ne lui sont attachés que d'un manière contingente. Il en ressort que le seul élément compris nécessairement

16. AT VIII, 46; A III, 156-157.

17. Margaret WIISON, entre autres, a proposé ce rapprochement (cf. Descartes, p. 82 et 83). Cependant, elle défend en même temps l'interprétation d'après laquelle l'essence des corps, pour Descartes, représente la somme de leurs propriétés invariantes. Il est clair que les deux interprétations sont incompatibles : Descartes pourrait admettre que le fait d'être coloré constitue une propriété permanente du morceau de cire mais soutenir en même temps qu'il est possible de concevoir un corps entièrement dépourvu de couleur, de sorte que la couleur ne serait pas une propriété constiturive du morceau de cire

18. AT VII, $31 ;$ A II, 424 
dans l'idée d'une chose matérielle est l'étendue: la proposition «Tout corps est étendu» énonce une vérité «analytique», donc nécessaire, à propos des corps, alors que «Tout corps a un poids (ou une certaine température) » énonce seulement à leur sujet une vérité (à supposer que ce soit bien une vérité) "synthétique » et contingente. ${ }^{19}$ L'extension, avec la flexibilité et la mutabilité, apparaît comme une propriété appartenant à l'essence du morceau de cire parce qu'elle est constitutive du concept d'une chose matérielle (elle ne pourrait pas en être «enlevée») et qu'il serait contradictoire de nier une "vérité conceptuelle» comme "Tout corps est étendu » alors qu'il n'y a aucune contradiction à concevoir un corps dénué d'un poids, d'une couleur ou d'une température. ${ }^{20}$

Je supposerai dans ce qui suit que l'«expérience de pensée» des Principes réussit à montrer que la nature des corps est l'étendue et que c'est à une telle «analyse» que Descartes fait allusion dans la seconde Méditation. Un autre point, plus difficile à expliquer, est la raison pour laquelle une connaissance claire et distincte des corps ne dépend pas de ce qui est donné «par l'entremise des sens». Pourquoi, comme il le dit apparemment dans le passage cité au début où il présente son « raisonnement»,

19. À propos des exemples des Principes, M. WILson écrit (Descartes, p. 83): «(...) it seems that Descartes' underlying purpose is really to get us to consider what belongs to our concept of body (...). Descartes is really asking what properties are analytically contained in the concept of body in general. "

20. On pourrait penser à une autre interprétation des passages des Principes. Descartes pourrait faire implicitement appel à un critère pouvant se formuler comme suit : «s'il existe un seul corps dénué d'une certaine propriété, alors celle-ci n'appartient pas à l'essence des choses matérielles. » Si en effet, comme on peut le supposer, tous les corps ont les mêmes propriétés essentielles (un morceau de cire, par exemple, doit être de même nature qu'un morceau de bois) et s'il y a un seul corps n'ayant pas une certaine propriéré, alors celle-ci n'est pas commune à tous les corps et, par conséquent, n'est pas une de leurs propriétés constitutives. Par exemple, certains corps peuvent être réduits en poudre ; donc la dureté n'est pas une propriété partagée par tous les corps et on peut l'exclure de leur essence. De même, s'il existe un seul corps parfaitement transparent et n'ayant aucune couleur (même si tous les autres sont colorés), alors on peut aussi exclure la couleur. (Ou bien encore: parce que le morceau de cire en se réchauffant a perdu son odeur, ou bien parce qu'il y a des corps sans odeur, et parce que certains corps comme le feu n'ont pas de pesanteur, l'odeur et la pesanteur ne font pas non plus partie de leur essence). Il n'existe par contre aucun corps n'ayant pas de grandeur ou de forme; ces deux propriétés sont absolument communes à tous les corps et, par conséquent, on peut les compter au nombre de leurs propriétés constitutives. La difficulté dans ce critère est que l'impossibilité de découvrir un corps dépourvu de relle ou telle propriété ne prouve évidemment pas que tous la possèdent. 
est-ce que ce ne sont pas les sens qui peuvent nous renseigner sur la nature des choses matérielles?

La raison pour laquelle ce ne sont pas les sens qui sont capables de donner une connaissance distincte des corps est que l'entendement ou l'esprit joue un rôle indispensable quand il s'agit de connaître l'essence de quelque chose. Dans un passage des Principes qui rappelle celui du Discours cité plus haut, on lit que «(...) ce ne sont point nos sens qui nous font découvrir la nature de quoi que ce soit, mais seulement notre raison lorsqu'elle y intervient (...). ${ }^{21}$ En quoi consiste plus exactement la fonction exercée par l'entendement ou la raison? On peut penser ici à plusieurs interprétations différentes. Une première interprétation, commune à beaucoup de commentateurs des Méditations, revient à affirmer que l'idée d'une chose étendue n'a pas été obtenue par les sens, qu'elle ne manifeste pas une propriété sensible des choses, parce qu'elle a son origine dans l'entendement lui-même. C'est ce que soutient apparemment Descartes dans les Principes (deuxième partie, fin de l'article 3) où il écrit : « (...) nous quitterons (...) tous les préjugés qui ne sont fondés que sur nos sens, et ne nous servirons que de notre entendement, parce que c'est en lui seul que les premières notions ou idées (...) se trouvent naturellement. \$ ${ }^{22}$ Immédiatement à la suite, on lit (début de l'article 4) le passage déjà cité : «En ce faisant, nous saurons que la nature (...) du corps pris en général (...) ne consiste point en ce qu'il est une chose dure, ou pesante, ou colorée, ou qui touche nos sens de quelque autre façon, mais seulement en ce qu'il est une substance étendue (...). . ${ }^{23}$ On peut donc savoir à l'aide de l'entendement seul, par les idées qui s'y trouvent «naturellement», par conséquent de façon innée, que la nature des corps est leur extension ; c'est pourquoi la connaissance de leur nature ne doit rien aux sens. ${ }^{24}$

Un seconde interprétation serait la suivante. Descartes veut peut-être mettre en lumière la différence existant entre un concept, en particulier ici le concept d'une chose étendue, qui est un produit

21. AT VIII, 37; A III, 142 .

22. AT VIII, $42 ;$ A III, 148

23. AT VIII, $42 ;$ A III, 149

24. Cette interprération est celle adoptée par exemple par Marrial Gueroult, Descartes selon l'ordre des raisons (Paris: Aubier, 1953), vol. I, p. 132 et suiv., et par M. WILSON, Descartes, p. 169 
de l'entendement, et des données des sens ou bien des images créées par l'imagination. Dans le troisième alinéa du passage du morceau de cire (dans l'original latin du texte, qui comprend en tout sept alinéas), il soutient que la possession du concept d'un corps ne consiste pas simplement dans le fait d'avoir des impressions sensibles ou des images dans l'esprit, parce que le concept, une représentation intellectuelle, donc non sensible, permet de penser une infinité de grandeurs possibles (pour l'extension) et de formes possibles (pour la flexibilité) alors que ni les sens, ni même l'imagination ne peuvent représenter une variété infinie de formes ou d'objets de grandeurs différentes: on peut par les sens ou l'imagination se représenter des objets de grandeurs et de formes déterminées, mais non pas un corps en général (l'extension et la flexibilité en elles-mêmes) sans aucune grandeur ou aucune forme particulière. L'entendement aurait par conséquent une fonction indispensable à remplir quand nous nous formons des concepts (des représentations non sensibles) des choses, et on pourrait soutenir en ce sens que c'est par l'entendement seul qu'il est possible de parvenir à une connaissance de quelque chose. ${ }^{25}$

Il y a encore une troisième interprétation qui pourrait être donnée de la remarque au sujet du rôle de l'entendement. « [Ce] ne sont point nos sens qui nous font découvrir la nature de quoi que ce soit, mais seulement notre raison lorsqu'elle y intervient ", parce que les sens peuvent donner seulement une idée obscure et confuse de la nature des choses matérielles et que c'est à la raison ou à l'entendement qu'il revient de rendre distincte cette représentation. L'، expérience de pensée » des Principes montre de quelle manière se forme l'idée claire et distincte de la substance étendue : l' " analyse » revient à retirer de l'idée d'un corps (quelconque) tout ce qui ne lui "appartient" par réellement, ou ce sans quoi il pourrait quand même exister, c'est-à-dire toutes les qualités qui, comme l'odeur, la couleur ou la température, en rendent la représentation obscure et confuse. L'idée du corps est confuse quand il s'y mêle des éléments qui ne lui sont pas essentiels et devient distincte quand elle ne comprend plus que ce qui lui appartient en propre. L'opération consistant à discerner les éléments qui appartiennent réellement à

25. Certe interprétation, si je le comprends bien, est celle de Bernard WiLliams; cf. Descartes: The Project of Pure Enquiry, p. 222 et 223. 
l'idée des corps (l'étendue, la flexibilité et la mutabilité) et à en retirer les éléments inessentiels est l'œuvre de l'entendement (et non des sens ou de l'imagination).

Il n'y a peut-être pas une preuve décisive que l'on pourrait apporter à l'appui de l'une ou l'autre de ces trois interprétations, mais c'est la troisième, il me semble, ou peut-être une combinaison de la deuxième et de la troisième, qui est la plus plausible.

D'après la première interprétation, Descartes veut introduire une distinction entre les propriétés sensibles des corps et leurs propriétés non sensibles: on connaît les propriétés sensibles par l'entremise des sens, tandis que les autres ont leur source dans l'entendement lui-même. Un premier point à noter à propos de cette interprétation est que la distinction ne découle pas de l'« expérience de pensée » des Principes. Celle-ci est censée montrer que les propriétés "sensibles» telles que la couleur, l'odeur ou la température doivent être exclues de l'essence des corps; mais il n'en découle pas que les propriétés qui restent, une fois retirées ces propriétés, devraient être des propriétés non sensibles ou que celles-ci devraient avoir leur origine dans l'entendement plutôt que dans les sens. On pourrait soutenir peut-être que la proposition «Tout corps est étendu » exprime une vérité nécessaire tandis que «Tout corps a un poids » exprime seulement à leur sujet une vérité contingente, mais il ne s'ensuit pas que l'idée d'extension devrait pour cette raison avoir une origine différente de celle de l'idée d'une chose ayant un poids (ou de l'idée de pesanteur).

En deuxième lieu, on peut se demander dans quelle mesure Descartes soutient réellement que l'idée d'étendue ne représente pas une propriété sensible des corps. Il semble y avoir après tout des passages dans lesquels il dit que nous pouvons percevoir, par l'intermédiaire des sens, des choses étendues (ayant une grandeur déterminée, une longueur, une forme, immobiles ou en mouvement). À l'article 3 déjà cité des Principes (deuxième partie), qui porte le titre: "Que nos sens ne nous enseignent pas la nature des choses, mais seulement ce en quoi elles nous sont utiles ou nuisibles », il écrit : «Il suffira que nous remarquions seulement que tout ce que nous a percevons par l'entremise de nos sens se rapporte à l'étroite union qu'a l'âme avec le corps, et que nous connaissons ordinairement par leur moyen ce en quoi les corps de dehors nous peuvent 
profiter ou nuire, mais non pas quelle est leur nature, si ce n'est peut-être rarement et par hasard. ${ }^{26} \mathrm{Ce}$ qui laisse entendre que nous pouvons au moins savoir par l'intermédiaire des sens que les corps ont telles ou telles dimensions, telles ou telles formes, et peut-être même aussi, quoique "rarement et par hasard", que l'étendue appartient à leur essence (contrairement d'ailleurs à ce que dit le titre de l'article). C'est ce que suggère aussi un passage de la sixième Méditation où on lit: «(...) au dehors [en ce qui concerne les choses extérieures] outre l'extension, les figures, les mouvements des corps, je remarquais en eux ["je sentais aussi en eux", dit plus précisément le texte latin : sentiebam etiam in illis] de la dureté, de la chaleur, et toutes les autres qualités qui tombent sous l'attouchement (...).» ${ }^{27}$ Il semble que d'après ce passage également on puisse percevoir par le moyen des données des sens les grandeurs, les formes et les mouvements des corps aussi bien que leurs couleurs, leurs poids ou leurs températures.

La principale difficulté dans la première interprétation, il me semble, est celle-ci. Descartes ne pourrait pas nier de façon plausible que seuls les sens peuvent nous informer sur les grandeurs ou les formes des choses. Mais si c'est empiriquement qu'il est possible de déterminer qu'un objet a telle ou telle longueur ou telle ou telle forme particulières, on ne voit pas en quel sens on pourrait dire que c'est à l'aide des idées contenues ( «naturellement ») dans l'entendement lui-même, et non au moyen des sens, que l'on peut savoir en général que les objets ont une grandeur (qu'ils sont étendus) ou qu'ils ont une forme. Il semble plutôt que, si on peut savoir au moyen des sens qu'un certain objet a une longueur $x$ ou $y$, alors on peut savoir aussi par le même moyen, quoiqu'à la condition peut-être que l'entendement y «intervienne», qu'il possède en général une longueur. En d'autres termes, on ne voit pas comment la propriété d'avoir une longueur pourrait être une propriété non sensible d'un corps, au sens où elle aurait son origine dans l'entendement lui-même, alors qu' « avoir une longueur de dix mètres » devrait être une propriété sensible de ce corps.

Il n'y a pas cette difficulté dans les deux autres interprétations. D'après la seconde, il n'est pas nécessaire de faire une distinction

26. AT VIII, 41-42; A III, 148.

27. AT VII, $74 ;$ A II, 484. 
entre les propriétés sensibles et les propriétés non sensibles dans la mesure où la fonction de l'entendement consiste simplement à former des concepts (des représentations intellectuelles et non sensibles) des choses telles que les sens les présentent, par exemple le concept d'une chose ayant une grandeur, ou bien le concept d'une chose colorée (ou d'une chose ayant une température, etc.). La troisième interprétation ne présuppose pas non plus une telle distinction: entre les propriétés constitutives et les propriétés accidentelles des corps (ou bien entre les idées claires et dictinctes et les idées obscures et confuses contenues dans leur représentation) la distinction n'est pas la même que celle entre des idées dont l'origine est intellectuelle et des idées ayant une origine sensible. Le rôle de l'entendement (la manière dont il «intervient»), d'après cette interprétation, est de rendre claire et distincte, en la décomposant en ses éléments, l'idée du morceau de cire telle que les sens l'ont donnée et renfermant aussi bien des qualités comme l'extension ou la flexibilité que des qualités comme la couleur, la température ou l'odeur. C'est apparemment ce qui est suggéré dans ce passage (fin du troisième alinéa) où on lit: «(..) la perception [de la cire] n'est pas une vision, ni un attouchement, ni une imagination (...), mais seulement une inspection de l'esprit, laquelle peut être imparfaite et confuse, comme elle était auparavant, ou bien claire et distincte, comme elle est à présent, selon que mon attention se porte plus ou moins aux choses qui sont en elles, et dont elle est composée." ${ }^{28}$ L'idée complexe du corps nous est donnée par l'intermédiaire des sens, et c'est par un acte de l'entendement que l'esprit dirige son attention vers les différents éléments contenus en elle pour en retirer les idées obscures et confuses et retenir celles qui sont claires et distinctes : un acte de l'entendement et, par suite, quelque chose que les sens seuls ne pourraient pas donner. ${ }^{29}$

28. AT VII, 31; A II, 426 (je souligne).

29. Cette interprétarion s'accorde avec celle que défend Robert MCRAE dans un article intitulé "Innate Ideas», R.J. Butler (ed.) Cartesian Studies (Oxford: Basil Blackwell, 1972). McRae écrit (p. 46): "Our idea of bodies as extended [selon Descartes / comes from bodies acting on us through the senses. Much that comes to us through the senses, however, is obscure. That is why at the beginning of Meditation $V$ Descartes' first cask was to separate out what is distinct in our ideas of body from what is obscure. " Mais cette séparation, il me semble, contrairement à ce que dit McRae, s'opère, implicitement au muins, dès la seconde Méditation, et elle est rappelée, cette fois de façon explicite, kł.ıns la troisième (dans un passage que je cite ci-dessous et dans lequel Descartes 
Une dernière remarque à propos du rôle de l'entendement. Il est probable que Descartes fasse allusion dans le passage du morceau de cire aux deux fonctions, l'une consistant à se former des concepts des choses et l'autre à isoler les idées claires dans la représentation des corps. Il semble néanmoins que la plus importante soit la seconde : ce n'est qu'en faisant la distinction entre ce qui est clair et ce qui est obscur dans les idées des choses matérielles que l'on peut arriver à découvrir leur essence. L'autre fonction ne permet pas de faire une distinction entre leurs propriétés constitutives et leurs propriétés accidentelles: on peut se former des concepts tels que celui d'extension ou de flexibilité, mais également des concepts comme «avoir une couleur » (ou bien "être blanc», ou "avoir l'odeur des fleurs») sans qu'on puisse en tirer une distinction entre les deux types de propriétés. Mais puisqu'il n'y a aucune incompatibilité entre les deux fonctions, on peut supposer qu'il pense ici autant à l'une qu'à l'autre. ${ }^{30}$

En débutant, j’ai cité le raisonnement par lequel Descartes concluait que, puisqu'il est possible que toutes les propriétés «sensibles» du morceau de cire changent pendant que celui-ci demeure, ce n'est pas par l'intermédiaire des sens que l'on peut parvenir à une connaissance distincte du corps. Il ne semble pas que le raisonnement se fonde sur une différence entre des propriétés "changeantes» et des propriétés «permanentes" des corps: quand on cherchè̀ savoir en quoi consiste la nature du morceau de

renvoie à la seconde Méditation). McRae ajoute ensuite (avec raison, je pense): "The distinction made berween clear and distinct ideas on the one hand, and obscure and confused on the orher, is not the same as that between innate ideas and adventitious ideas, or ideas derived from senses. The later distinction is one between two kinds of ideas according to their origin. The former distinction is based on differences of degree, and can be found within all perceprion, whether the ideas involved are adventitious or innate. The work of artending and distinguishing whereby what we perceive is made clear and distinct is the work of the understanding as opposed to the senses, but this work of the understanding can equally well be directed at eirher the innate or the adventitious». (ibid).

30. Ce qui précède n épuise évidemment pas la question de l'innéisme de Descartes. Cf. sur ce point l'article de R. MCRAE, "Innate Ideas ». McRae ne se réfère pas ici au texte de la seconde Méditation. Pourtanr le passage du morceau de cire, comme je le montre dans ce qui suit, est compatible avec ses conclusions et, au moins en partie, les confirment. 
cire, on doit déterminer non pas quelles sont ses propriétés permanentes mais quelles sont celles qui appartiennent en général à l'idée d'une chose matérielle (les idées claires qu'elle renferme) ou bien, ce qui revient peut-être au même, on doit découvrir quelles propriétés ont en commun le morceau de cire et tous les autres corps. D'autre part, en faisant une distinction entre leurs propriétés essentielles et leurs propriétés accidentelles, il ne semble pas qu'il pense à une opposition entre des propriétés connues exclusivement à l'aide de l'entendement et des propriétés perçues au moyen des sens (comme plus haut dans la première des trois interprétations mentionnées). Pourtant, n'est-ce pas précisément ce qui est suggéré par le raisonnement? Pour citer à nouveau le texte, on lit : "Qu'est-ce donc que l'on connaissait en ce morceau de cire avec tant de distinction ? Certes ce ne peut être rien de tout ce que j'y ai remarqué par l'entremise des sens (...). " ${ }^{31} \mathrm{Il}$ est clair d'après ce passage qu'il y a pour lui quelque chose que l'on connaît distinctement dans le morceau de cire qui n'est pas donné par les sens et qui peut être connu exclusivement à l'aide de l'entendement. Mais ce quelque chose, je pense, ce n'est pas l'essence ou en général les propriétés des corps mais leur substantialité. ${ }^{32}$

Dans la troisième Méditation, Descartes dresse la liste des idées claires et distinctes contenues dans la représentation de la matière ; il mentionne entre autres la substance : « (...) si [i']examine [les choses matérielles] de la même façon que j'examinais (...) l'idée de la cire, je trouve qu'il ne s'y rencontre que fort peu de choses que je conçoive clairement et distinctement, à savoir la grandeur ou bien l'extension (...); la figure qui est formée par les termes et les bornes de cette extension ; la situation (...); et le mouvement ou le changement de cette situation; auxquelles on peut ajouter la substance, la durée, et le nombre. ${ }^{33}$ Quand il demande dans la deuxième Méditation ce que l'on connaissait

31. AT VII, 30; A II, 424 (je souligne).

32. Jean-Marie Btyssalt: avance cette interprétation, mais sans la développer, dans un article intitulé "L'analyse du morceau de cire. Contribution à l'étude des "degrés du sens" dans la Seconde Méditation de Descartes », H. Waciner (brsg.) Sinnlichkeit und Verstand in der deutschen und französischen Pbilosophie von Descartes bis Hegel (Bonn : Bouvier, 1976), en particulier p. 15 à 19. B. Wil.Llams également la mentionne (Descartes: The Project of Pure Enquiry, p. 220 et 221 ) et la discure brièvement (cf. cidessous note 46).

33. AT VII, 4.3; A II, 442 
"avec tant de distinction" dans le morceau de cire, il est donc possible qu'il pense non à l'extension du corps (ou bien à sa "situation", à son "nombre", ou à sa "durée") mais plutôt au corps conçu comme une substance.

Par ailleurs, le morceau de cire est décrit comme quelque chose qui «demeure ", après avoir été approché du feu, alors que ses propriétés ont toutes changé. Descartes emploie ici le même verbe trois fois (remanere et non superesse comme dans l'alinéa suivant); il écrit: "La même cire demeure-t-elle [remanetne] après ce changement? Il faut avouer qu'elle demeure [remanere], et personne ne le peut nier. (...) toutes les choses qui tombaient [sous le sens] se trouvent changées, et cependant la même cire demeure [remanet]. ${ }^{34}$ Une interprétation au moins plausible serait de supposer que ce qui dans le corps a demeuré à travers le changement qu'ont subi ses propriétés, c'est la chose elle-même considérée comme une substance ; ce qui a demeuré, ce ne sont pas ses propriétés "permanentes" par opposition à ses propriétés "changeantes», mais la substance même par opposition à ses propriétés.

Un autre passage (cité en partie précédemment) qui donne à penser que Descartes songe ici à la substantialité des corps est le suivant : «Peut-être [ce qui est connu distinctement] était ce que je pense maintenant, à savoir que la cire n'était pas, ni cette douceur du miel, ni cette agréable odeur des fleurs, ni cette blancheur, ni cette figure, ni ce son, mais seulement un corps qui un peu auparavant me paraissait sous ces formes [modis], et qui maintenant se fait remarquer sous d'autres. » ${ }^{35} \mathrm{D}$ 'après l'interprétation courante, on doit paraphraser ce texte comme suit: ce que l'on connaît distinctement dans le morceau de cire, ce ne sont pas ses propriétés sensibles comme l'odeur, la blancheur, le son, etc., lesquelles ont toutes changé, mais ses propriétés « non sensibles » et invariantes : son étendue, sa flexibilité et sa mutabilité, c'est-à-dire ces qualités auxquelles on reconnaît qu'il s'agit d'un corps (quelque chose de matériel). Mais on peut donner une autre interprétation de ce passage. On doit se rappeler d'abord la définition de la substance telle qu'elle est énoncée (par exemple) dans les Principes : «Lorsque

34. AT VII, 30; A Il, 424.

35. AT VII, 30-31; A II, 424. 
nous concevons la substance, nous concevons seulement une chose qui existe en telle façon qu'elle n'a besoin que de soi-même pour exister. » ${ }^{36}$ Par contraste, on appelle " propriétés » (" qualités » ou "modes", ou encore "attributs") ces choses qui dépendent de quelque chose d'autre pour exister (à savoir une certaine substance, à laquelle elles sont inhérentes). Comme il le dit parfois, on doit distinguer entre, d'un côté, les « choses subsistantes » et, de l'autre, les "dépendances 》 de ces choses, c'est-à-dire leurs modes, leurs propriétés ou accidents. Dans ce passage de la seconde Méditation, il soutient qu'on ne conçoit pas distinctement le morceau de cire si on le considère comme une collection ou un ensemble de qualités (la blancheur, une certaine forme, un son particulier, une odeur) et si on ne rapporte pas ces qualités ou ces modes à la chose dont ils dépendent. Dans les Principes, à cet endroit où il traite de la distinction modale, il écrit : « (...) nous pouvons apercevoir clairement la substance sans [un de ses modes], mais (...) réciproquement nous ne pouvons avoir une idée distincte [d'un mode] sans penser à une telle substance. ${ }^{37}$ Il fait ici une observation similaire. On a une conception adéquate (distincte) des propriétés du morceau de cire quand on les conçoit comme des modes d'une certaine substance et non comme des choses qui pourraient en être séparées et qui pourraient subsister par elles-mêmes (comme si elles étaient des substances). Et on a une idée distincte du morceau de cire quand on le conçoit non pas comme "cette douceur du miel, (...) cette agréable odeur des fleurs, (...) cette blancheur, [ou] cette figure », c'est-à-dire comme une simple collection de propriétés, mais plutôt comme une chose (une substance) qui auparavant se manifestait par certaines propriétés (ou modes) et qui maintenant en possède d'autres. ${ }^{38}$

Pour revenir au raisonnement, comment l'interpréter si c'est de la substantialité des corps qu'il est question dans tous ces

36. AT VIII, 24; A III, 122.

37. AT VIII, 29; A III, 130.

38. Il fait une remarque similaire à un autre endroit dans les Principes. Il écrit : « (...) quand nous (...) considérons [les modes] comme les propriétés des substances dont [ils] dépendent, nous les distinguons aisément de ces substances, et les prenons pour [tels qu'ils | sont véritablement; au lieu que si nous voulions les considérer sans substance, cela pourrait être cause que nous les prendrions pour des choses qui subsistent d'ellesmêmes; en sorte que nous confondrions l'idée que nous devons avoir de la substance avec celle que nous devons avoir de ses propriétés. "(AT VIII, 31 ; A III, 134). 
rextes? Je pense que l'on pourrait maintenant formuler comme suit sa conclusion: "la perception distincte du morceau de cire conçu comme une substance ne dépend en rien de ce qui nous est donné par l'intermédiaire des sens. »Et la prémisse pourrait se formuler: "toutes les propriétés (particulières) du corps qui tombent sous les sens ont changé » ou, ce qui revient au même, «toutes les propriétés (particulières) du corps ont changé », puisque, d'après ce qui a été dit plus haut, toute propriété d'un corps est une propriété sensible. Le raisonnement pourrait alors s'interpréter de la façon suivante: "Toutes les propriétés du corps ont changé (du moins il était possible que toutes changent) quand celui-ci a été approché du feu. Pourtant, nous gardons une conception du morceau de cire comme de quelque chose qui a demeuré à travers ce changement; nous le concevons comme le porteur de toutes ces propriétés changeantes. Donc, si les propriétés ont changé, cette conception que nous avons du corps, comme le substrat permanent de ses propriétés, nous ne l'avons pas obtenue au moyens des sens ; elle doit par conséquent avoir sa source dans l'esprit lui-même. »

Descartes, à ma connaissance, n'invoque nulle part ailleurs cet argument pour montrer que la conception du morceau de cire, quand on le considère comme une substance, comme ce qui demeure à travers ses propriétés changeantes, doit avoir son origine dans l'entendement. Néanmoins, il y a des endroits, entre autres dans les Principes, où il fait une remarque, apparentée il me semble, qui pourrait aider à mieux comprendre le sens du raisonnement : la remarque d'après laquelle on sait en vertu d'une sorte de principe connu a priori (par la « lumière naturelle ») que, là où se trouvent des propriétés ou des accidents, il doit exister une certaine substance. Il écrit par exemple : «(..) il est manifeste, par une lumière qui est naturellement en nos âmes, que le néant n'a aucunes qualités ni propriétés qui lui soient affectées, et qu'où nous en apercevons quelques-unes $i l$ se doit trouver nécessairement une chose ou substance dont elles dépendent. » ${ }^{39}$ De façon similaire, dans un autre article des Principes: «(...) l'une de nos notions communes est que le néant ne peut avoir aucuns attributs, ni propriétés ou qualités: c'est pourquoi, lorsqu'on en rencontre quelqu'un, on a raison de conclure qu'il est l'attribut de quelque

39. AT VIII, 8 ; A III, 97 (je souligne). 
substance, et que cette substance existe. ${ }^{40}$ En effet, comme il le remarque, on ne peut avoir une connaissance «immédiate» des substances; par exemple, dans les quatrièmes Réponses: «(...) nous ne connaissons point les substances immédiatement par elles-mêmes; mais, de ce que nous apercevons quelques formes, ou attributs, qui doivent être attachés à quelque chose pour exister, nous appelons du nom de Substance cette chose à laquelle ils sont attachés. " ${ }^{41}$ De ces passages ont peut déduire qu'une substance ne peut être connue « immédiatement » en ce sens qu'il est nécessaire d'inférer son existence de la perception de ses propriétés, et que c'est une maxime ou un principe a priori de la raison (quelque chose qui est révélé par le seul entendement) qui autorise à faire l'inférence, c'est-à-dire le principe ou la "notion commune» d'après laquelle là où on trouve des propriétés (des attributs ou des modes), il doit exister une substance à laquelle elles sont inhérentes.

On pourrait faire un rapprochement avec le raisonnement de Descartes. Il est probable qu'il existe pour lui d'autres vérités connues par la « lumière naturelle » concernant l'idée de substantialité ayant un statut semblable à celle d'après laquelle toute propriété doit être rattachée à une substance. On peut penser en particulier au principe revenant à affirmer que tout changement suppose l'existence d'une substance dans laquelle a lieu ce changement. I1 semble en effet que pour lui tout changement doive être nécessairement un changement se produisant dans les propriétés d'une chose ; les substances elles-mêmes, qui constituent le substrat du changement, ne peuvent changer, c'est-à-dire disparaître ou cesser d'exister, car, dit-il, elles sont « de leur nature incorruptibles. » ${ }^{42} \mathrm{Il}$ est plausible de supposer qu'il accepte ce principe comme une autre vérité connue par le seul entendement, le principe, d'ailleurs traditionnel comme le premier, d'après lequel, lorsqu'on perçoit un changement, "on a raison de conclure» qu'il existe une substance dans laquelle il se produit. Et on pourrait comprendre maintenant le raisonnement comme suit : «Supposons que pendant un court instant non seulement le morceau de cire mais tous les autres objets perçus subissent un changement radical et continuel

40. AT VIII, 25; A III, 123 (je souligne).

41. AT VII, $222 ;$ A II, 662.

42. AT VII, $14 ; A$ II, 401 . 
de leurs propriétés : la table sur laquelle est posé le morceau de cire change graduellement de forme, de volume, de couleur, de poids; les murs de la pièce où se trouve la table changent de longueurs et de hauteurs ; même notre propre corps change toutes ses propriétés. Les objets familiers disparaissent tous au cours du changement et il devient impossible de distinguer la table du morceau de cire, les murs du plancher, ou notre corps du siège sur lequel nous sommes assis. Pourtant, malgré ces changements et au moment même où ils se produisent, nous sommes certains que quelque chose demeure ("personne ne le peut nier»). Mais ce ne sont pas les sens qui peuvent nous assurer de l'existence de ce quelque chose qui demeure puisque (par hypothèse) il ne se présente plus rien de stable ou de constant dans les impressions reçues des sens et que tout ce que nous pouvons percevoir, ce sont des propriétés en un changement incessant. » Descartes peut ensuite conclure : «Si ce n'est pas par les sens que dans une telle situation nous pourrions savoir que quelque chose demeure, alors c'est au moyen de notre seul entendement. Nous savons qu'il y a quelque chose qui demeure par-delà tous ces changements parce que nous nous appuyons sur le principe, qui se trouve "naturellement " dans notre esprit, d'après lequel tout changement suppose l'existence d'une substance comme substrat de ce changement. »

Descartes pourrait ne pas faire une distinction entre ce dernier principe et celui mentionné précédemment : entre «Tout changement (de propriétés) suppose une substance en laquelle il se produit " et «Une propriété suppose toujours l'existence d'une substance à laquelle elle est rattachée. " Il considère peut-être que le premier principe est compris dans le deuxième (au sens où il en représenterait un cas particulier). Mais l'important est que pour lui notre connaissance de l'existence des substances est toujours conclue de la perception d'une quelconque de leurs propriétés, ou de la perception d'un changement, et que cette connaissance prend appui sur un principe (une "notion commune ») de notre raison. Elle n'est pas conclue au sens où on pourrait inférer seulement de façon probable l'existence des substances de celle de leurs propriétés ; il s'agit au contraire d'une connaissance certaine, même si elle est "médiate", dans la mesure où elle dépend d'un principe de la raison. Et c'est, je pense, le sens du raisonnement : la connaissance (de l'existence) des substances est conclue de la perception des 
propriétés, ou de changements de propriétés, mais elle ne provient pas des sens; aucune impression sensible ne peut en elle-même prouver la présence d'une substance (on ne peut pas littéralement "percevoir» par la moyen des sens des choses subsistantes). Pour employer un langage qui fait évidemment penser à Kant, la connaissance (de l'existence) des substances représente plutôt la “contribution a priori» de l'entendement à notre connaissance des choses: nous croyons "voir» des substances, mais c'est en réalité notre entendement qui nous fait conclure qu'elles existent.

Pour revenir au texte, les quatrième et cinquième alinéas du passage (toujours dans le texte latin) confirment, assez clairement il me semble, cette interprétation. Dans le troisième alinéa, Descartes était parvenu à la conclusion (dans un passage en partie cité plus haut) que « (...) [la] perception [de la cire] (...) n'est point une vision, ni un attouchement, ni une imagination, et ne l'a jamais été, quoiqu'il le semblât ainsi auparavant, mais seulement une inspection de l'esprit (...). » Dans le quatrième, il poursuit en remarquant que, sur ce point, « [nous sommes] presque trompé[s] par les termes du langage ordinaire. » Que disons-nous dans le langage ordinaire? Il écrit : « Nous disons (...) que nous voyons la cire elle-même [ipsammet] si elle est présente [si adsit], et non pas que nous jugeons par sa couleur ou sa figure [ex colore vel figura] qu'elle est présente [eam adesse]. D'où je pourrais tout de suite conclure : je connais la cire par une vision des yeux, non par une inspection de l'esprit seul. ${ }^{43}$ On pourrait rendre plus explicite le texte comme suit. Nous croyons savoir que le morceau de cire est présent (qu'il existe) par une vision des yeux alors qu'en fait nous jugeons (inférons ou concluons), de ce que nous remarquons certaines propriétés (une couleur ou une figure), que la chose (une substance) est présente. Parce que nous percevons ces propriétés, nous disons dans le langage ordinaire que nous connaissons par les sens l'existence du morceau de cire; en réalité, nous jugeons, en partant de cette perception, qu' il existe une substance à laquelle ces

43. AT VII, 32 ; A II, 426 (trad. mod.). La traduction du duc de Luynes, pleine de contresens, se lit comme suit: "Car nous disons que nous voyons la même cire si on nous la présente, et non pas que nous jugeons que c'est la même, de ce qu' elle a même couleur et même figure; d'où je voudrais presque conclure, que l'on connaît la cire par la vision des yeux, et non par la seule inspection de l'esprit." 
propriétés sont inhérentes à l'aide du principe suivant lequel toute propriété suppose une substance à laquelle elle est rattachée. Dans le même alinéa, pour illustrer la même idée, Descartes suggère une analogie entre la connaissance du morceau de cire et celle que nous avons de ces hommes que nous voyons marcher dans la rue et dont nous n'apercevons que les chapeaux et les manteaux. Nous disons dans le langage ordinaire que nous "voyons" ces hommes tout comme nous disons «voir» le morceau de cire. Ce que nous voyons, en réalité, ce ne sont que des chapeaux et des manteaux, et nous jugeons (concluons), de la vision de ces vêtements, que ce sont des hommes. Il en est de même pour la connaissance des corps : « je comprends par la seule puissance de juger qui réside en mon esprit, ce que je croyais voir de mes yeux. $\gg{ }^{44}$ Pendant que nous examinons le morceau de cire, nous observons une certaine forme, une couleur, une odeur, nous entendons un son, nous avons la sensation de quelque chose de froid au toucher et, à partir de ces impressions, nous jugeons qu'un corps est "présent». Nous concluons (la raison mène à conclure) qu'il y a une chose, une certaine substance, à laquelle doivent être rapportées toutes ces propriétés.

Le cinquième alinéa n'ajoute aucune idée nouvelle sauf peutêtre sa dernière phrase, qui peut sembler un peu obscure. On lit : "Mais quand je distingue la cire d'avec les formes extérieures, et que tout de même que si je lui avais ôté ses vêtements, je la considère toute nue, certes quoiqu'il se puisse encore rencontrer quelque erreur dans mon jugement, je ne la puis concevoir de certe sorte sans un esprit humain. " ${ }^{45} \mathrm{Il}$ serait difficile de rendre compte du sens de ce texte (comme d'ailleurs de l'alinéa qui précède) dans l'interprétation habituelle du passage. On pourrait se demander quels sont ces «vêtements » ou ces «formes extérieures» et que pourrait vouloir dire "considérer tout nu» le morceau de cire suivant cette interprétation (peut-être les "vêtements "sont-ils les propriétés sensibles ou non permanentes du corps, et que considérer "tout nu» le morceau de cire serait le considérer seulement comme quelque chose d'étendu, de flexible et de muable ; ce qui néanmoins n'apparaît pas très plausible). Si je l'interprète

44. AT VII, 32; A II $; 427$.

45. AT VII, 32; A II, 427-428. 
correctement, les «formes extérieures » ou les «vêtements » du morceau de cire (qui font penser à ceux des hommes dans la rue de l'alinéa précédent), ce sont ses propriétés ou ses accidents, et quand on considère celui-ci «tout nu» en le distinguant de ces vêtements ou de ces formes, on le considère simplement comme une substance en l'opposant à ses propriétés, lesquelles sont des «dépendances » de cette substance comme les vêtements à l'égard de la personne qui les porte. Pour Descartes, il est vrai, comme je l'ai noté, on ne peut connaître «immédiatement » une substance sans aucune propriété (ce que pourrait suggérer, faussement, l'expression "considérer tout nu» le morceau de cire): on sait qu'une substance existe parce qu'on en remarque des propriétés, et connaître une substance (savoir quelque chose à son sujet), c'est connaître ses propriétés (puisque c'est par celles-ci seulement qu'elle se manifeste). Mais, et c'est ce qu'il veut dire dans ce cinquième alinéa, on peut concevoir le morceau de cire comme distinct et indépendant de ses accidents, c'est-à-dire comme quelque chose qui peut se manifester parfois sous telles ou telles « formes extérieures » et d'autres fois sous d'autres formes, et, selon lui, on a besoin d'un esprit humain pour en avoir une telle conception (l'inverse n'étant pas vrai : comme il a été noté plus haut, on n'aurait pas selon lui une conception adéquate d'un accident ou d'un mode si on le concevait comme quelque chose pouvant subsister indépendamment d'une substance). ${ }^{46}$

46. Comme je l'ai noté, B. WiL.IIAMs, dans son commentaire du passage du morceau de cire, mentionne, pour la rejeter ensuite, une interprétation du type de celle défendue ici. Mais le sens de son objection n'est pas très clair. Il écrit (Descartes: The Project of Pure Enquiry. p. 221) : "If Descartes's whole metaphysical interest (dans ce passage) is in distinguishing substance from properties, and he is concerned with isolating the substance which has or supports the properties, how can he be satisfied with ending up, as he does, with a characterization of the wax which is irself entirely in terms of properties - the properties of being extended, flexible and changeable? » En quel sens est-ce que l'on peut dire que Descartes en vient à caractériser le morceau de cire uniquement "en termes de propriétés "? Il rejette sûrement, d’après ce qui précède, le point de vue d'après lequel une substance se réduir à (n'est rien d'autre que) la somme de ses artributs (on ne connait, certes, les substances que "médiatement ", par le biais de leurs artributs, ce qui n'empêche pas que l'on sache, par la "lumière naturelle", qu'elles existent et qu'elles diffèrent de leurs propriétés). Mais Williams poursuit en écrivant (ibid.) : « $\lfloor\mathbf{I t}\rceil$ is Descartes's general doctrine that a substance is only "by a distinction of reason" different from its essential attributes, and that one makes the nature of a substance known by listing its essential attributes (...). .) La remarque, semble-t-il, porte plus précisément sur la question des attriburs essentiels. La difficulté (si je le comprend bien) serait que, puisque pour Descartes ce n'est que par une 
L'interprétation défendue jusqu' ici diffère de l'interprétation courante en particulier sur deux points. (a) En ce qui concerne son essence, on peut dire que l'entendement, selon Descartes, a un rôle à jouer dans la connaissance du morceau de cire non pas au sens où il serait lui-même la source, entièrement «a priori», de l'idée d'étendue, mais en ce sens que seul l'entendement peut discerner dans l'idée sensible du morceau de cire ce qui appartient en propre aux corps (c'est-à-dire leurs propriétés non-accidentelles); et en ce sens également que c'est l'entendement qui a pour fonction de former des concepts des choses (en particulier le concept de quelque chose d'étendu, de flexible et de muable). (b) Pour ce qui concerne la substantialité des corps, par contre, il semble que l'idée d'une substance (ou la distinction entre substance et accidents) ne soit pas selon lui issue des sens et ait effectivement son origine ( "naturellement») dans l'entendement. Il existe, au sujet de la substantialité, des principes ou "notions communes ", c'est-à-dire des « vérité[s] éternelle[s] qui [ont leur] siège en notre pensée » ${ }^{47}$, entre autres le principe d'après lequel une propriété est toujours rattachée à une substance, ou le principe qui veut qu'un changement soit nécessairement un changement se produisant dans les propriétés d'une chose (j'aurai l'occasion plus loin de mentionner un autre de ces principes). Bref, l'entendement exerce incontestablement un rôle important dans la connaissance des corps, mais on ne décrit pas correctement ce rôle dans l'interprétation courante du texte.

\section{III}

J'ai donné jusqu'ici les grandes lignes d'une interprétation des cinq premiers alinéas. On pourrait la résumer, brièvement, comme suit. Le premier alinéa formule l'objection, dont il était question au

abstraction que l'on peut concevoir une substance sans un des deux attributs essentiels (la pensée ou l'étendue), la conséquence serait qu'il ne peut justifier la distinction, pourtant centrale dans son système, entre substance er propriétés (car on ne trouve pas cette asymétrie, selon Williams, dans le cas des attributs essentiels, que l'on trouve entre substances et propriétés). À cela, je pense, on peut répondre simplement que, à supposer qu'il y ait en effet une telle difficulté dans la distinction faite par Descartes, il ne sensuit pas (on commettrait un non sequitur de croire qu'il s'ensuit) que la distinction ne joue pas un rôle fondamental dans son système.

47. AT VIII, 23; A III, 120. 
débur, à laquelle le passage a pour but de répondre (j'y reviens cidessous). Ensuite, dans le deuxième, Descartes présente son raisonnement qui tend à montrer que l'idée distincte du morceau de cire conçu comme une substance trouve son origine dans la seule faculté de penser et non dans les sens. Le troisième apparaît plus complexe : au début, il y est encore question du corps considéré comme une substance (comme quelque chose se manifestant par le biais de ses modes) et, ensuite, comme une chose étendue (une substance matérielle). On peut noter que cet alinéa est le seul dans lequel Descartes mentionne l'essence des corps (à cet endroit où il remarque que ce qui reste quand on éloigne « toutes les choses qui n'appartiennent point à la cire » est leur extension, leur flexibilité et leur mutabilité ${ }^{48}$ ). C'est dans ce troisième alinéa également qu'il fait allusion aux différentes « fonctions » assumées par l'entendement dans la connaissance des corps. Dans le quatrième et le cinquième, finalement, il reprend à nouveau l'idée de substantialité pour souligner le rôle de l'entendement dans notre conception distincte du morceau de cire. Il me reste à donner une interprétation des deux derniers alinéas, le sixième et le septième. Ici également la notion de substance va se révéler la notion-clé du texte.

Descartes reprend en ce point l'objection citée en débutant et qui donnait sa raison d'être au passage; il s'agissait de la difficulté soulevée à propos de la possibilité d'une connaissance du moi pensant. Le sixième alinéa commence par une question: «Mais enfin que dirai-je de cet esprit, c'est-à-dire de moi-même [ ?] (...) [Que] prononcerai-je (...) de moi qui semble concevoir avec tant de netteté et de distinction ce morceau de cire?» Et il poursuit: « $[\mathrm{Ne}]$ me connais-je pas moi-même, non seulement avec bien plus de vérité et de certitude, mais encore avec beaucoup plus de

48. Je ne nie pas l'importance de la remarque de Descartes au sujet de l'essence des corps (mais elle devient apparente surtour dans les Méditations suivantes), pas plus que sa pertinence quand on la considère seulement dans le cadre de cette seconde Méditation. On pourrait dire qu'elle est pertinente pour les deux raisons suivantes: (1) Si le passage du morceau de cire décrit les divers éléments clairs et distincts que comporte la représentation des corps (dont leur substantialité, qui est ici le plus important), on peut comprendre que Descartes veuille mentionner aussi l'idée d'étendue comme étant un de ces éléments. (2) Puisqu'il est question du rôle de l'entendement dans la conception des corps comme substances, il est normal qu'il parle également dans ce contexte des autres fonctions de l'entendement, en particulier celle consistant à décomposer l'idée d'un corps et à en isoler les propriétés essentielles. 
distinction et de netteté ? ${ }^{49}$ Si au début la connaissance du moi, quand on la comparait à celle des corps, pouvait paraître douteuse et problématique, il semble maintenant que l'inverse plutôt soit vrai : c'est l'esprit qui va apparaître à la fin comme étant " mieux connu» que les corps. Il s'agit de savoir comment Descartes en arrive à cette conclusion.

L'objection était celle-ci. Il y a une difficulté dans l'idée d'une connaissance du moi parce que le moi n'est pas quelque chose qui tombe sous le sens ou que l'on peut se rendre présent par des images au moyen de l'imagination, alors qu'on estime ordinairement que toute connaissance doit débuter par le témoignage des sens ou s'y rapporter au moins indirectement. Autrement dit, puisque l'esprit ( «cette je ne sais quelle partie de moi-même ») n'est pas le type de chose qui est susceptible d'être vu ou touché, on peut se demander dans quelle mesure et comment il est possible de le connaître. Pour répondre à l'objection, Descartes doit montrer qu'il peut y avoir une connaissance qui ne dépend en aucune façon des données sensibles, c'est-à-dire montrer comment est possible une connaissance d'une «chose» qui ne se présente jamais aux sens.

Cependant, on peut deviner, en lisant entre les lignes, une autre objection à laquelle il pense aussi ou, peut-être, une autre façon de formuler la même objection. Dans l'alinéa qui précède immédiatement le passage du morceau de cire, il rappelait pourquoi on doit considérer le moi comme une substance (une res) et les pensées comme ses propriétés. Le moi, disait-il, est une chose « qui doute, qui conçoit, qui affirme, qui nie, qui veut, qui ne veut pas, qui imagine aussi, et qui sent. » Toutes ces pensées «appartiennent " (pertineant) au moi de la même façon que des propriétés sont dites appartenir à la chose à laquelle elles sont rattachées. Une pensée, en effet, n'est pas une chose susceptible d'une existence séparée du moi. On lit, dans le même alinéa : «Y a-t-il [aucune de ces choses : douter, concevoir, désirer, nier] qui puisse être distingué[e] de ma pensée, ou qu'on puisse dire être séparé[e] de moi-même? » Non, puisqu' « il est de soi si évident que c'est moi qui doute, qui entends, et qui désire, qu'il n'est pas ici besoin de rien ajouter pour

49. AT VII, 32-33; A II ; 428 . 
l'expliquer. " ${ }^{50}$ On ne pourrait pas concevoir un doute ou un désir sans un moi qui doute ou désire pas plus que la blancheur sans une chose à laquelle soit inhérente cette blancheur. Il faut donc considérer les pensées comme des «dépendances» du moi de la même façon qu'une certaine forme, une odeur, une couleur particulière doivent être regardées comme des «dépendances»du morceau de cire. Mais qui pourrait vouloir nier que le moi est une "chose» (une substance) et les pensées des accidents ou des «dépendances» de cette chose? Un philosophe, dans la tradition scolastique, qui voit dans le moi (ou l'âme) non pas une substance mais une «forme», en l'occurrence la « forme substantielle» d'un corps d'être humain (vivant). Pour un philosophe de «l'École», une substance, normalement, doit être un corps, une chose matérielle, et il considère le moi ou l'esprit comme étant rattaché à ce corps comme une propriété l'est à une substance, alors que seul le corps lui-même (celui de l'individu humain) peut être regardé véritablement comme une chose capable de subsister par elle-même. Il semble que ce soit à une telle façon de voir qu'il est fait allusion dans un passage des Principes où on lit: «(...) pendant les premières années de notre vie nous nous [sommes] si fort accoutumés à sentir et imaginer, que nous [avons] acquis une facilité plus grande à penser de cette sorte [en employant nos sens et notre imagination]; de là vient que beaucoup de personnes ne sauraient croire qu'il y ait de substance si elle n'est imaginable et corporelle, et même sensible ; (...) de là vient aussi que la plupart du monde se persuade qu'il n'y a rien qui puisse subsister [subsistere] si ce n'est un corps $(\ldots) .{ }^{51}$

Si c'est à une telle façon de voir que Descartes fait allusion dans le passage du morceau de cire, il semble qu'une objection à laquelle il essaie de répondre soit la suivante : « toute substance est un corps, c'est-à-dire une chose dont on a (ou au moins peut avoir) connaissance par l'intermédiaire des sens ou se forme des images par l'imagination; comme l'esprit n'est pas quelque chose dont on peut avoir une telle connaissance, alors ce n'est pas une substance.» Le fait qu'il pense à une telle objection est très nettement confirmé par la manière dont il y répond dans le sixième alinéa. La réponse s'appuie sur les remarques qu'il faisait auparavant à propos de la

50. AT VII, $29 ;$ A II, 421 .

5I. AT VIII, 37 ; A III, 142 (trad. mod.). 
substantialité du morceau de cire; il écrit : «[Est-ce que je ne me connais pas moi-même | non seulement avec bien plus de vérité et de netteté [que je ne connais le morceau de cire], mais encore avec beaucoup plus de distinction et de netteté ? Car si je juge que la cire (...) existe, de ce que je la vois, certes il suit bien plus évidemment que (...) j'existe moi-même, de ce que je la vois. " 52 Il affirme ici deux choses. (a) Nous jugeons (ou concluons) que le morceau de cire existe parce que nous le « voyons »; plus exactement, de ce que nous appréhendons par les sens une de ses propriétés, par exemple sa forme ou sa couleur, nous concluons (jugeons) qu'il existe : nous concluons de l'existence d'une de ses propriétés à celle de la chose à laquelle elle est inhérente. De la même façon, nous voyons le morceau de cire (ou remarquons une de ses propriétés), et cette vision du morceau de cire est une pensée et, partant de cette pensée, nous concluons que nous existons à titre de substance. Le principe sur lequel nous nous appuyons est le même et dans les deux cas nous jugeons ou concluons qu'une certain substance existe, à savoir le morceau de cire ou le moi pensant. (b) D'autre part, de ce que nous avons cette pensée particulière (cette vision de la cire), nous concluons que nous existons comme substance " plus évidemment» qu'il ne suit, de la perception de l'une de ses propriétés, que le morceau de cire existe comme substance. Pourquoi "plus évidemment "? La raison est qu'en ce point des Méditations l'existence des choses matérielles n'est pas certaine, parce qu'elle tombe toujours sous le coup du doute de la première Méditation (qui sera levé seulement dans la sixième), alors qu'au début de cette seconde Méditation l'existence du moi a été établie, de façon définitive, par le Cogito. Immédiatement après, on lit : «Car il se peut faire que ce que je vois ne soit pas en effet de la cire, il peut aussi arriver que je n'aie pas même des yeux pour voir aucune chose; mais il ne se peut pas faire que lorsque je vois [...ou] pense voir, que moi qui pense ne sois quelque chose [non aliquid sim ]. " ${ }^{53}$ Bref, on est certain que le moi existe comme substance (qu'il est "quelque chose"), mais on ne sait pas encore avec certitude s'il existe des corps, des substances dont l'essence réside dans l'étendue.

52. AT VII, 33; A II, 428.

53. Ibid. 
On peut voir maintenant que Descartes veut répondre en réalité à deux objections différentes quoique liées: (1) Le moi pensant n'est pas une substance, parce que seules des choses

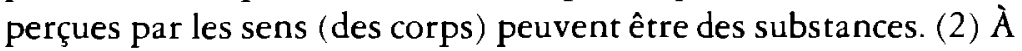
supposer même qu'il soit une substance, il ne serait pas possible de savoir quoi que ce soit à son sujet puisqu'il n'est pas quelque chose susceptible d'être vu ou touché. La réponse à la première objection consiste simplement à rappeler comment on peut établir qu'une substance existe : il suffit que l'on remarque une certaine propriété, comme « avoir une forme cylindrique » ou bien « avoir l'odeur des fleurs », d'où on conclut qu'il y a une substance à laquelle elle est rattachée. Mais il n'est pas nécessaire que celle-ci soit une propriété sensible comme "avoir une forme cylindrique»; il est possible aussi que ce soit une propriété telle que "percevoir l'odeur des fleurs», ou bien "penser au cercle carré», ou "imaginer un triangle rectangle.» On sait également qu'il y a une chose à laquelle ces propriétés sont inhérentes, de sorte qu'on peut savoir que le moi est une substance. Comme on le lisait dans le quatrième alinéa, si le morceau de cire n'est pas quelque chose que l'on peut «voir» (si on ne peut «voir» au moyen des sens qu'il est une substance), et si la substantialité des choses est toujours « conclue " (elle dépend d'un jugement de l'entendement), alors il n'y a aucune difficulté à admettre que le moi, que l'on ne peut pas non plus «voir», soit aussi une substance.

La réponse à la seconde objection revient à dire ceci : le fait qu'il ne soit pas une chose visible, tangible ou audible n'empêche pas qu'on puisse connaître l'esprit, puisque la "connaissance» d'une chose n'est rien d'autre que la connaissance de ses propriétés, et il est clair qu'il existe un très grand nombre de propriétés que l'on attribue couramment au moi dans la mesure où chacune des pensées représente une de ses propriétés. Par conséquent, il n'y a pas non plus de difficulté dans l'idée d'une connaissance de l'esprit même s'il n'est pas quelque chose qui peut se représenter par les sens.

Par ailleurs, non seulement une connaissance du moi est possible, Descartes soutient également que celui-ci est mieux connu et qu'il est connu plus distinctement que les corps. Il écrit, toujours dans le sixième alinéa : si « (...) la connaissance de la cire semble être plus nette et plus distincte, après qu'elle a été découverte 
non seulement par la vue, ou par l'attouchement, mais encore par beaucoup d'autres causes [en l'occurrence par les idées de l'entendement lui-même] : avec combien plus (...) de distinction (...) me dois-je connaître moi-même. » Il explique ensuite en quel sens : d'une part, « (...) toutes les raisons qui servent à connaître la cire, ou quelque autre corps, prouvent beaucoup plus (...) évidemment la nature de mon esprit. " ${ }^{54}$ En effet, à chacune des propriétés attribuées aux corps correspond potentiellement une propriété de l'esprit ; si « avoir l'odeur des fleurs ", par exemple, représente une propriété d'un certain corps, «sentir l'odeur des fleurs (que possède ce corps) », doit être regardée, en tant que pensée, comme une propriété de l'esprit (et chacune de ces propriétés le fait connaître). D'autre part, « (...) il se rencontre encore tant d'autres choses en l'esprit lui-même, qui peuvent contribuer à l'éclaircissement de sa nature, que celles qui dépendent du corps, comme celles-ci, ne méritent quasi pas d'être nombrées. »Car il y a plusieurs propriétés de l'esprit qui ne correspondent à aucune des propriétés des corps (par exemple, «juger que le théorème de Pythagore est vrai » ou " penser au cercle carré »), de sorte qu'il y a au total beaucoup plus de propriétés qui peuvent être attribuées à l'esprit qu'il y en a qui peuvent être attribuées aux corps, puisque l'esprit possède non seulement ces propriétés auxquelles correspondent des qualités des corps, mais aussi celles qui «se rencontrent [seulement] en l'esprit lui-même» (comme la propriété de "penser au cercle carré »). Descartes fait ici appel implicitement à un autre principe (ou notion commune), à propos du concept de substantialité, le principe que plus on connaît de propriétés d'une substance, plus et mieux on la connaît. Suivant ce principe, parce qu'il y a un plus grand nombre de propriétés que l'on peut attribuer à l'esprit, on en a une connaissance meilleure que celle que l'on peut avoir des corps.

Je citerai à l'appui de cette interprétation un dernier passage tiré des Principes (première partie) : l'article 11, intitulé « Comment nous pouvons plus clairement connaître notre âme que notre corps ». Le contenu de cet article reprend très exactement le contenu du sixième alinéa, mais sous une forme beaucoup plus explicite. Descartes écrit d'abord : « (...) afin de savoir comment la

54. AT VII, 33; A II, 428-429 (trad. mod.). 
connaissance que nous avons de notre esprit précède celle que nous avons du corps, et qu'elle est incomparablement plus évidente, (...) nous remarquerons qu'il est manifeste, par une lumière qui est naturellement en nos âmes, que le néant n'a aucunes qualités ni propriétés qui lui soient affectées, et qu'où nous en apercevons quelques unes il se doit trouver nécessairement une chose ou substance dont elles dépendent. " ${ }^{55}$ Immédiatement à la suite, il cite l'autre principe, concernant la notion de substantialité, qui vient d'être mentionné : «Cette même lumière nous montre aussi que nous connaissons d'autant mieux une chose ou substance, que nous remarquons en elle davantage de propriétés (...). » ${ }^{56}$ Ces deux principes permettent d'expliquer en quel sens l'esprit est mieux connu que les corps. Par le second principe, on peut dire que l'esprit est mieux connu parce qu'on en connaît plus de propriétés : «(...) il est certain que nous en remarquons beaucoup plus en notre pensée qu'en aucune autre chose, d'autant qu'il n'y a rien [par exemple des propriétés d'un corps] qui nous excite à connaître quoi que ce soit, qui ne nous porte encore plus certainement à connaître notre esprit.» Le premier principe, d'un autre côté, permet d'affirmer que l'esprit est une substance à laquelle doivent être rapportées toutes ces propriétés; on lit: «(...) si je me persuade qu'il y a une terre à cause que je la touche ou que je la vois : de cela même, par une raison encore plus forte, je dois être persuadé que mon esprit existe (...).» Car il se pourrait "que je pense toucher la terre, encore qu'il n'y ait peut-être aucune terre au monde ; cependant il n'est pas possible que mon esprit ne soit rien [ ibil sit] pendant qu'il a cette pensée (...). ${ }^{57}$

Ce passage des Principes montre de façon particulièrement claire de quelle manière Descartes répond aux deux objections. D'une part, certains philosophes doutent que le moi soit une chose subsistante. ${ }^{58}$ On peut répondre à cela que la substantialité du moi

55. AT VIII, $8 ;$ A III, 97.

56. Ibid.

57. AT VIII, 8-9; AT III, 97-98 (trad. mod.).

58. Il est intéressant de noter que l'objection est très proche de celle que Kant, d'ailleurs à la suite de Hume, va adresser à Descartes dans sa critique du "paralogisme de la substantialité ». Cf. Critique de la raison pure (trad. de A. Tremesaygues et B. Pacaud, Paris: Presses Universitaires de France, 1968), en particulier p. 284. Er Traité de la nature bumaine (trad. de A. Leroy, Paris : Aubier, 1946), p. 342 et suiv. On pourrait dire en ce sens que Descartes répond par avance à cette objection que feront les deux philosophes. 
découle simplement du principe a priori (une vérité enseignée par la «lumière naturelle») d'après lequel une propriété est nécessairement rattachée à une certaine substance : s'il y a des pensées, il doit exister une substance qui possède ces pensées. D'autre part, à quelqu'un qui émet des doutes quant à la possibilité d'une connaissance de l'esprit (quelque chose qui ne tombe pas sous les sens), la réponse est que toute pensée, considérée comme une propriété, nous fait connaître l'esprit (et une pensée n'est pas quelque chose dont on a connaissance en ayant recours aux sens). On peut même dire, par le deuxième principe, que l'esprit est «mieux connu » que les corps dans la mesure où on en connaît plus de propriétés: même après que l'existence des choses matérielles aura été démontrée, il y aura toujours un plus grand nombre de propriétés que l'on pourra attribuer à l'esprit, aussi bien les pensées qu'il trouve « en lui-même » que toutes ces autres pensées ayant pour objets des corps. Bref, Descartes peut écarter l'objection d'après laquelle une chose qui ne se présente jamais aux sens n'existe pas (à titre de substance) et l'objection d'après laquelle, même si elle existait, elle serait strictement inconnaissable. Il s'ensuit qu'il faut rejeter les deux thèses soutenues par les scolastiques: que seuls les corps peuvent être qualifiés de choses subsistantes, et que toute connaissance doit nécessairement débuter par des données des sens.

Le septième alinéa rappelle, pour terminer, quelle leçon on doit tirer, au sujer de la connaissance de l'esprit, des considérations sur la connaissance des corps. On lit : « (...) puisque c'est une chose qui m'est à présent connue, qu'à proprement parler nous ne concevons les corps que par la faculté d'entendre qui est en nous (...), et que nous ne les connaissons pas de ce que nous les voyons, ou que nous les touchons, mais seulement de ce que nous les concevons par la pensée, je connais évidemment qu'il n'y a rien qui me soit plus facile [facilius aut evidentius] à connaître que mon esprit. " ${ }^{99}$ Descartes décrit, de manière extrêmement concise, le résultat de l'ensemble du passage. L'exemple du morceau de cire était destiné à mettre en lumière l'existence d'un «élément $a$ priori » qui intervient nécessairement dans toute connaissance des corps, à savoir la conception que l'on a des corps comme des substances. On sait a priori, par la seule faculté de penser, (a) que là 
où on trouve des propriétés, il doit exister une substance; $(b)$ que celle-ci se manifeste par le biais de ces propriétés (et c'est par elles seulement qu'il est possible de la connaître); (c) et, par conséquent, que plus on en remarque de propriétés, mieux on la connaît. Si c'est là, au moins en partie, ce que suppose la conception distincte du morceau de cire, on peut comprendre que la connaissance du moi soit aussi "facile et manifeste " et même "meilleure » que celle des corps dans la mesure où elle fait intervenir le même "élément a priori» que celui qui rend possible la conception discincte des choses matérielles, avec cette différence que la connaissance de l'esprit n'a pas besoin d'avoir recours au témoignage des sens (puisque quelqu'un ne connaît pas ses pensées au moyen des sens), contrairement à la connaissance d'un corps (un corps particulier comme un morceau de cire), laquelle dépend autant des données sensibles que de cette «contribution a priori » de l'entendement. On peut donc dire qu'il est plus «facile» à quelqu'un de connaître son propre esprit que n'importe quelle autre chose puisqu'il suffit qu'il ait des pensées pour qu'il soit certain d'exister comme substance et qu'il se connaisse lui-même (en ayant connaissance de ses propriétés que sont ces pensées) alors même qu'il ne sait pas, comme dans cette seconde Méditation, si les corps existent et si des propriétés qu'il croit observer (une certaine forme, une certaine couleur) représentent réellement des propriétés de quelque chose. ${ }^{60}$

Pour conclure, l'interprétation que j'ai voulu défendre dans ce qui précède pourrait se résumer en trois points. (1) D'après la lecture courante du passage, Descartes veut mettre ici surtout en lumière le fait que les corps soient des choses étendues : au début,

60. Si on sait que le moi pensant existe comme substance parce qu'on en connaît des propriétés (ì savoir ses pensées), on pourrait se demander pourquoi il ne serait pas aussi certain, en vertu du même principe a priori, que les corps, dont on connaît également des propriétés, existent comme substances, donc existent tout court. Fst-ce qu'il ne devraic pas y avoir d'après ce qui précède une symétrie complète entre le cas du moi er celui de la substance matérielle? Descartes répundrait ceci: le passage du morceau de cire décrit la conception que l'on a du moi et des corps, une conception telle quion doir nécessairement se les représenter comme des choses subsistantes; mais dans la seconde Méditatıon seule l'existence du moi (comme substance) est certaine, ayant été prouvée pur le Cogito. On est amené par le même principe a priori à se représenter le moi pensint $e t$ les corps comme des substances, mais c'est dans une autre Méditation que sera démontrée la validité du principe : dans la présente Méditation, on peut tenir pour certaine, en conséquence du Cogito, uniquement la substantialité du moi. 
dans la première partie de la Méditation, il a décrit l'esprit comme une chose dont la nature est de penser, et il passe ensuite, dans la deuxième, à l'idée que nous nous faisons de l'essence des corps (à savoir l'idée de choses étendues, flexibles et muables). Mais si mon interprétation du texte est exacte, c'est l'ensemble de la seconde Méditation et non seulement sa première partie qui traite de l'esprit : Descartes a montré d'abord, dans la première, que le moi existe comme une substance par nature pensante et, dans la deuxième, il répond à des objections qui pourraient être opposées à cette conception de l'esprit et aux doutes sur la possibilité de le connaître. (2) D'autre part, son but premier en introduisant l'exemple du morceau de cire est de montrer non pas que l'essence des corps se trouve dans l'étendue, ou que l'idée des corps comme choses étendues dépend, exclusivement ou non, de l'entendement, mais que sans l'«intervention» de l'entendement (sans sa "contribution a priori») ceux-ci ne pourraient être conçus comme des substances. Il y a en effet dans le passage un seul alinéa portant (en partie) sur l'essence des corps (à cet endroit où il fait allusion à une "expérience de pensée " comme celle que décrivent les Principes) et la remarque à ce sujet, replacée dans le contexte de la Méditation, apparaît seulement comme une remarque incidente : toutes ses autres considérations à propos du morceau de cire, à l'exception de celles portant sur ce que j'ai appelé les différentes "fonctions » de l'entendement, ont trait à la substantialité des corps. (3) Finalement, et c'est le point le plus important, le rôle de l'exemple du morceau de cire est subordonné au but ultime du passage qui est de montrer que le moi pensant est une substance (une res) au même sens où les corps sont qualifiés de substances et, bien qu'il ne soit pas susceptible d'être représenté par les sens ou à l'aide de l'imagination, qu'il est possible d'en avoir connaissance et qu'il est «mieux connu» que les corps.

Département de philosophie

CEEGP de Saint-Hyacinthe 